\title{
Noninvasive Assessment of the Circle of Willis in Cerebral Ischemia: The Potential of CT Angiography and Contrast-Enhanced Transcranial Color-Coded Duplexsonography
}

\author{
G. Gahn ${ }^{\mathrm{a}}$ J. Gerber ${ }^{\mathrm{b}}$ S. Hallmeyer ${ }^{\mathrm{a}}$ H. Reichmann ${ }^{\mathrm{a}}$ R. von Kummer ${ }^{\mathrm{b}}$ \\ Departments of a Neurology and ${ }^{b}$ Neuroradiology, University of Dresden, Germany
}

\section{Key Words}

Stroke $\cdot$ CT angiography $\cdot$ Ultrasound $\cdot$ Ultrasound contrast agent $\cdot$ Transcranial color-coded

duplexsonography

\begin{abstract}
Thirty-four patients with acute hemispheric ischemic strokes underwent both CT angiography and contrastenhanced transcranial color-coded duplexsonography (TCCD) to study the effectiveness of the combined noninvasive techniques for evaluation of the circle of Willis. In 3/34 patients, CT angiography and contrast-enhanced TCCD demonstrated middle cerebral artery (MCA) occlusion, in 5 others MCA stenosis. A severe posterior cerebral artery stenosis was missed by CT angiography. In 8 patients, contrast-enhanced TCCD failed because of poor bone windows. In these patients, CT angiography was normal. CT angiography and contrast-enhanced TCCD are complementary noninvasive diagnostic tools. Disagreements between the diagnostic findings of these methods still need further evaluation by digital subtraction angiography.
\end{abstract}

\section{Introduction}

Several studies have demonstrated the potential benefit of thrombolytic therapy in patients with acute cerebral ischemia [1-3]. There are increasing controversies about the need for demonstration of intracranial arterial occlusion before initiating thrombolytic therapy [4]. Since intra-arterial thrombolytic therapy greatly limits the broad applicability of this therapy, there is need for studying the cranial vasculature in a noninvasive fashion.

CT angiography has recently been found to be effective for evaluation of the intracranial arteries in acute stroke patients $[5,6]$. This technique tracks a bolus of contrast material after its intravenous injection through the cerebral vasculature by using continuous table motion through the CT gantry as the X-ray tube spins continuously. Most cerebral CT angiography studies use shaded-surface display as a postprocessing technique, thereby providing a three-dimensional vascular survey of the intracranial artery circulation. A drawback of this method is that it requires a threshold, creating a binary data set. The data below the threshold are not displayed, and no distinction is made between bone, intraluminal contrast enhancement and/or mural calcification above the threshold. An alternative postprocessing technique is maximum intensity projection which is not threshold dependent and

Georg Gahn, MD

Department of Neurology, Technical University of Dresden

Fetscher Strasse 74, D-01307 Dresden (Gemany)

Tel. +49 3514583565 , Fax +49 3514584365

E-Mail gahn@rcs.urz.tu-dresden.de
Fax +41613061234 E-Mail karger@karger.ch www. karger.com (c) 1999 S. Karger AG, Basel
$1015-9770 / 99 / 0095-0290 \$ 17.50 / 0$

Accessible online at: http://BioMedNet.com/karger 
preserves the $\mathrm{CT}$ attenuation values $[5,6]$. In both techniques, measurement of residual arterial lumen diameter by CT angiography, however, is limited by operatordepending offline data processing, especially in severe stenosis $[7,8]$. Several factors influence the feasibility of CT angiography in the acute stroke setting. Timing is critical, dilution of the contrast agent through the heart chamber implies dependence on stroke volume, and heat loading limits use of the X-ray tube [9]. In addition, CT angiography is contraindicated in patients with renal insufficiency, hyperthyroidism or known allergy against iodinated contrast agents and can therefore not be applied to all patients. Animal studies have shown that nonionic contrast agents do not increase the volume of ischemic infarction [10].

Transcranial color-coded duplexsonography (TCCD) is a relatively new diagnostic tool for noninvasive evaluation of the intracranial arteries. TCCD provides twodimensional data of the basal cerebral arteries [11]. Its usefulness is based on signal characteristics for vessel encoding and detection of turbulence rather than on an ability to provide a true morphologic picture of the vessel [9]. TCCD provides a real-time two-dimensional data set in one imaging plane requiring an experienced examiner for effective evaluation of the basal cerebral arteries [12]. TCCD examination of the cerebral vessels might be impossible due to alterations in the bony structure of the temporal bone. These alterations are found increasingly with elder age and in about $30 \%$ of stroke in aged patients $[13,14]$. One approach to overcome this limitation is the use of ultrasound contrast agents. Levovist ${ }^{\circledR}$ (Schering, Berlin, Germany) is the most widely investigated agent and has recently been registered for the German market. It has been shown to be effective for the evaluation of the intracranial arteries in stroke patients with limited bone windows [15].

We evaluated the combination of CT angiography and contrast-enhanced TCCD to reduce the need for digital subtraction angiography (DSA) as far as possible. As a rationale we presumed that if both methods, $\mathrm{CT}$ angiography based on morphological data and contrast-enhanced TCCD based on physiological data, demonstrated identical findings, these results could be accepted as true.

\section{Materials and Methods}

During the period from January 1997 to April 1998 we saw 171 patients admitted to our Neurology department for transient or permanent ischemic hemispheric symptoms. Both contrast-enhanced TCCD and CT angiography were performed on thirty-four patients
Table 1. Number of patients with CT angiography (CTA), DSA and MR angiography (MRA) in addition to contrast-enhanced TCCD

\begin{tabular}{lc}
\hline Imaging study & Patients \\
\hline CTA & 27 \\
CTA + DSA & 4 \\
CTA + MRA & 2 \\
CTA + MRA + DSA & 1 \\
\hline
\end{tabular}

(12 males/22 females, mean age $69.6 \pm 10.9$ years). All patients were selected after demonstrating limited acoustical bone windows on nonenhanced TCCD and lack of contraindications against CT angiography. Mean time interval between onset of cerebral ischemia and CT angiography or contrast-enhanced TCCD was $8.1 \pm 6.6$ days. Contrast-enhanced TCCD and CT angiography were performed within $24 \mathrm{~h}$. Four patients had an additional DSA, 3 for preoperative workup of carotid artery stenosis, 1 because of progressive stroke (DSA confirmed normal contrast-enhanced TCCD and normal CT angiography findings). Two patients had additional MR angiography, 1 patient had both DSA and MR angiography (table 1).

\section{CT Angiography}

CT angiographies were performed by neuroradiologists (J.G., R.v.K.) with a Philips Gyroscan following the suggestions of Knauth et al. [5]. Patients were scanned from the floor of the sella up toward the vertex. The following scan parameters were used in all patients: 2:1 pitch, $1.5 \mathrm{~mm}$ collimation, $130 \mathrm{kV}$ and $125 \mathrm{~mA}$. Total scanning

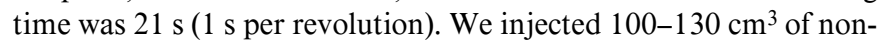
ionic intravenous contrast medium with an injection rate of $4 \mathrm{ml} / \mathrm{s}$ using an injection pump. Scan delay was determined by preceding bolus tracking. Three-dimensional reconstructions were performed using a surface-shaded rendering algorithm.

\section{TCCD Examination}

TCCD was performed by one person (G.G.) according to a protocol established by Bogdahn et al. [12]. As a contrast-enhancing agent, we used $2.5 \mathrm{~g}$ Levovist (Schering) in a concentration of $300-400 \mathrm{mg} /$ $\mathrm{ml}$. Depending on the duration of enhancement, up to four vials containing $2.5 \mathrm{~g}$ Levovist were injected. The examiner tried to identify all branches of the circle of Willis including the MCA with the origins of the M2 segments, the anterior cerebral artery (ACA) with the A2 segments and the posterior cerebral artery (PCA) with the P2 segments as well as the distal intracranial segment of the internal carotid artery. Color flow signals were analyzed according to flow direction and according to turbulent flow which was further evaluated by duplex. These results were documented right after the exam in a selfdeveloped chart. For diagnosis of intracranial arterial stenosis, we followed the suggestions of Hennerici et al. [16]. TCCD examinations were performed with an Acuson $128 \mathrm{XP} / 4$ with a $2.0 / 2.5-\mathrm{MHz}$ transcranial sector scanner device.

Contrast-enhanced TCCD and CT angiography were performed and read blinded to the results of the corresponding imaging study, but with knowledge of the clinical symptoms. 

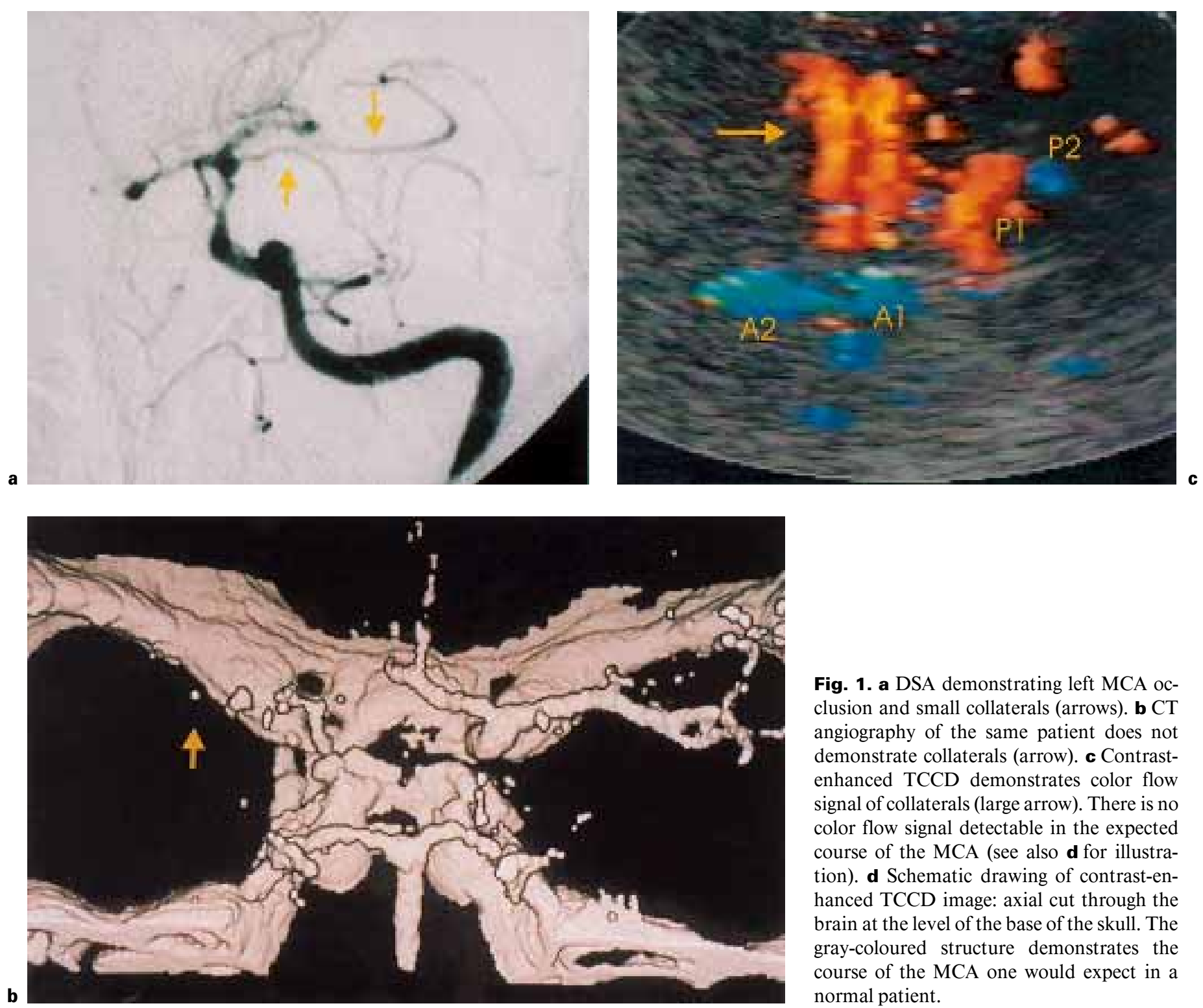

Fig. 1. a DSA demonstrating left MCA occlusion and small collaterals (arrows). b CT angiography of the same patient does not demonstrate collaterals (arrow). c Contrastenhanced TCCD demonstrates color flow signal of collaterals (large arrow). There is no color flow signal detectable in the expected course of the MCA (see also d for illustration). d Schematic drawing of contrast-enhanced TCCD image: axial cut through the brain at the level of the base of the skull. The gray-coloured structure demonstrates the course of the MCA one would expect in a normal patient.

\section{Results}

In 16 patients, both CT angiography and contrastenhanced TCCD demonstrated a normal circle of Willis. CT angiography and contrast-enhanced TCCD demonstrated occlusion of the MCA in 3 patients, in one of whom contrast-enhanced TCCD was able to detect the flow through small collaterals which were missed by CT angiography but confirmed by DSA (fig. 1). CT angiography and contrast-enhanced TCCD demonstrated five
MCA stenoses. One distal stenosis in the supraclinoid segment of the internal carotid artery was misdiagnosed by contrast-enhanced TCCD as a proximal MCA stenosis. One PCA stenosis by contrast-enhanced TCCD could not be confirmed by either CT angiography or DSA. This patient had recurrent complex focal seizures which might have contributed to a locally increased blood flow in the PCA. One severe stenosis of the PCA demonstrated by contrast-enhanced TCCD (systolic peak velocity $400 \mathrm{~cm} /$ s) in the P1 segment was first misdiagnosed by CT angiog- 


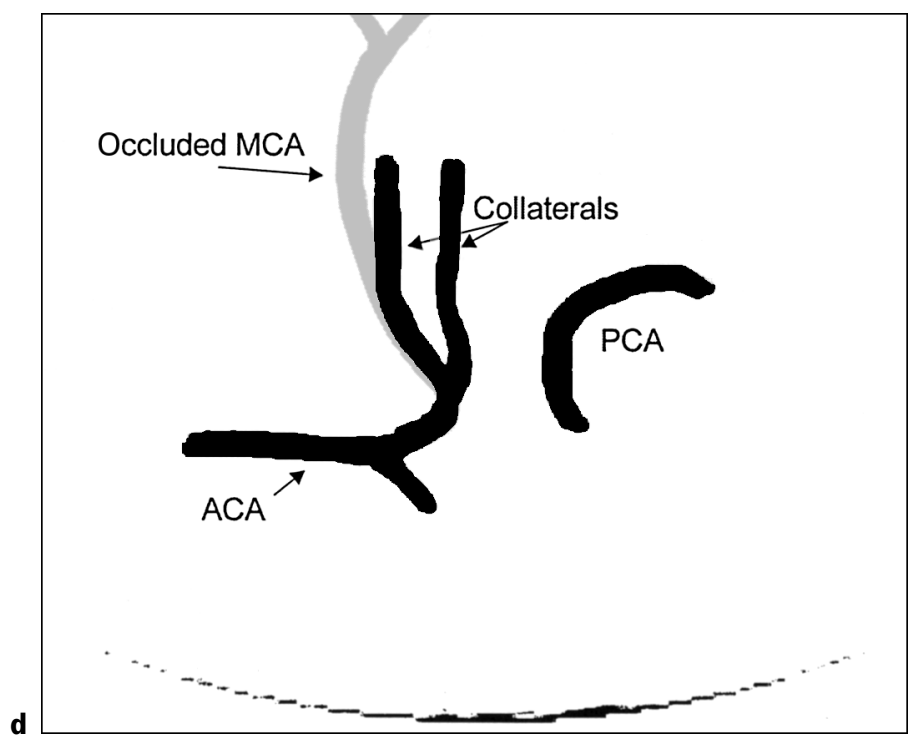

raphy as an aplasia of the $\mathrm{P} 1$ segment; by second review of the data in retrospective, a short segment of missing contrast filling in the P1 segment with a filled contrast column before and behind this region was detected. This was not consistent with a vessel aplasia, but with a severe stenosis. In 1 patient with unilateral internal carotid artery occlusion, an ipsilateral orthograd ACA was seen on contrast-enhanced TCCD and confirmed by DSA. In 1 patient with a hairline stenosis of the left internal carotid artery, contrast-enhanced TCCD demonstrated collateral flow through the anterior communicating artery, the direction of flow, however, could not be assessed by CT angiography.

Contrast-enhanced TCCD provided complete visualization of the circle of Willis in $24 / 34$ patients $(70.6 \%)$ without detectable color flow signals in unenhanced TCCD. The mean duration of ultrasound contrast enhancement was $2 \min 39 \mathrm{~s}$, with a range of $45 \mathrm{~s}$ to $7 \mathrm{~min}$ $6 \mathrm{~s}$. The enhancement started after a mean time of $22 \mathrm{~s}$ (range 6-37 s). In 3 patients, contrast-enhanced TCCD demonstrated only the ipsilateral part of the circle of Willis through one temporal window, there was no detectable signal from the other side. In 7 patients, however, no reasonable signal was detectable by contrast-enhanced TCCD. In all these 10 patients, CT angiography was normal.

\section{Discussion}

Because of the increasing application of systemic thrombolysis in acute stroke patients, there is need for noninvasive imaging of the cerebral vessels [4]. All currently available noninvasive imaging techniques are limited by several factors and do not reach either the sensitivity or the specificity of the gold standard DSA. However, there is a considerable risk of permanent neurological sequelae with DSA and it is not as widely available as CT [17]. MR angiography is relatively unspecific in detecting abnormalities of the cerebral vessels and needs further investigations $[9,18]$. We evaluated the effectiveness of combined CT angiography and contrast-enhanced TCCD for the noninvasive diagnosis of intracranial vessel abnormalities in patients with hemispheric ischemic strokes. The combination of both morphologically based CT angiography data and physiologically based TCCD data may improve the overall effectiveness of these two imaging techniques and obviate DSA for the diagnosis of cerebral vessel abnormalities.

CT angiography was able to demonstrate normal cerebral arteries as well as occlusion and moderate stenosis of the MCA. However, it was not able to reliably detect highgrade stenosis with a very small residual lumen diameter, neither could it detect small vessels with very low blood flow. Both these limitations could be overcome by contrast-enhanced TCCD; mild stenosis of the MCA, which tended to be overlooked by the film readers, could also be detected. Three-dimensional reconstruction of the CT angiography data set is operator dependent. Doing this procedure with the knowledge of ultrasound findings adds confidence to the CT angiography findings. This is confirmed by the CT angiography findings of 2 patients. In 1 patient, the reader of the CT angiograms first misdiagnosed a short segment of missing contrast agent in the P1 segment of the PCA as a vessel aplasia. After second review of the films with knowledge of the results from contrast-enhanced TCCD, demonstrating very high blood flow velocities in this segment, this was interpreted as a severe stenosis with a residual lumen diameter below the resolution of CT angiography. In a second patient with MCA occlusion, in whom contrast-enhanced TCCD detected flow through small collaterals (fig. 1), repeated reconstruction of CT angiography in retrospective also demonstrated these vessels.

Flow direction cannot be assessed by CT angiography, this was only possible by contrast-enhanced TCCD because of color flow encoding [19]. In patients with severe carotid disease, the demonstration of collateral pathways

Cerebrovasc Dis 1999;9:290-294 293 
through the circle of Willis provides important information for the patient's management.

Early evaluation of stroke patients for vascular abnormalities demonstrated an incidence of intracranial vascular occlusion of more than $50 \%[5,6,20]$. We evaluated the patients later during a normal hospital course (mean time interval between onset of ischemia and CT angiography and contrast-enhanced TCCD was 8.1 days) and found MCA occlusion in only $8.9 \%$ of our patients. In $17.6 \%$ of our patients, however, we found intracranial vascular stenosis which may reflect recanalization of primarily occluded vessels. We did not evaluate our patients in the hyperacute phase of cerebral ischemia. Our findings, however, suggest that both CT angiography and contrast-enhanced TCCD can provide important informa- tion about the cerebral vasculature during the very early ischemic phase. Both techniques can identify MCA occlusion as shown in 3 of our patients. Evaluation of more distal arterial branches, however, might be less reliable.

In conclusion, CT angiography and contrast-enhanced TCCD provide complementary information about the arteries of the circle of Willis. Disagreements between these two methods should be clarified by DSA.

\section{Acknowledgment}

This work has been supported by Schering, Berlin, Germany. This work has been presented in parts on the European Stroke Conference, Edinburgh, UK, 1998.

\section{References}

1 The National Institute of Neurological Disorders and Stroke rt-PA Stroke Study Group: Tissue plasminogen activator for acute ischemic stroke. N Engl J Med 1995;333:1581-1587.

2 Hacke W, Kaste M, Fieschi C, Toni D, Lesaffre E, von Kummer R, et al: Intravenous thrombolysis with recombinant tissue plasminogen activator for acute hemispheric stroke. The European Cooperative Acute Stroke Study (ECASS). JAMA 1995;274:1017-1025.

3 von Kummer R, Holle R, Rosin L, Forsting M, Hacke W: Does arterial recanalization improve outcome in carotid territory stroke? Stroke 1995;26:581-587.

4 Easton JD: Thrombolysis for stroke. Cerebrovasc Dis 1998;8:191-197.

5 Knauth M, von Kummer R, Jansen O, Hahnel S, Dorfler A, Sartor K: Potential of CT angiography in acute ischemic stroke. Am J Neuroradiol 1997; 18:1001-1010.

6 Wildermuth S, Knauth M, Brandt T, Winter R, Sartor K, Hacke W: Role of CT angiography in patient selection for thrombolytic therapy in acute hemispheric stroke. Stroke 1998;29:935938.

7 Lev MH, Ackerman RH, Lustrin ES, Brown JH, Gahn G, Chehade R: Procedure for accurate spiral $\mathrm{CT}$ angiographic measurement of luminal diameter (abstract). Radiology 1995; 197:S133.
8 Wong KS, Lam WW, Liang E, Huang YN, Chan YL, Kay R: Variability of magnetic resonance angiography and computed tomography angiography in grading middle cerebral artery stenosis. Stroke 1996;27:1084-1087.

9 Brant-Zawadzki M. Heiserman JE: The roles of MR angiography, CT angiography, and sonography in vascular imaging of the head and neck. Am J Neuroradiol 1997; 18:1820-1825.

10 Doerfler A, Engelhorn T, von Kummer R, Weber J, Knauth M, Heiland S, Sartor K, Forsting $\mathrm{M}$ : Are iodinated contrast agents detrimental in acute cerebral ischemia? An experimental study in rats. Radiology 1998;206:211-217.

11 Bogdahn U, Becker G, Schlief R, Reddig J, Hassel W: Contrast-enhanced transcranial color-coded real-time sonography. Results of a phase-two study. Stroke 1993;24:676-684.

12 Bogdahn U, Becker G, Winkler J, Greiner K, Perez J, Meurers B: Transcranial color-coded real-time sonography in adults. Stroke 1990;21: 1680-1688.

13 Itoh T, Matsumoto M, Handa N, Maeda $\mathrm{H}$ Hougaku H, Hashimoto $\mathrm{H}$, et al: Rate of successful recording of blood flow signals in the middle cerebral artery using transcranial Doppler sonography. Stroke 1993;24:11921195.

14 Seidel G, Kaps M, Gerriets T: Potential and limitations of transcranial color-coded sonography in stroke patients. Stroke 1995;26:20612066.
15 Gahn G, Elsner E, von Kummer R, Reichmann $\mathrm{H}$ : Constrast-enhanced transcranial color coded duplexsonography in acute stroke patients with limited bone windows (abstract). Neurology 1998;50(suppl 4):A76.

16 Hennerici M, Rautenberg W, Schwartz A: Transcranial doppler ultrasound for the assessment of intracranial arterial flow velocity Part 2. Surg Neurol 1987;27:523-532.

17 Heiserman JE, Dean BL, Hodak JA, Flom RA, Bird CR, Drayer BP, Fram EK: Neurologic complications of cerebral angiography. Am J Neuroradiol 1994;15:1401-1407.

18 Korogi Y, Takahashi M, Nakagawa T, Mabuchi N, Watabe T, Shiokawa Y, et al: Intracranial vascular stenosis and occlusion: MR angiographic findings. Am J Neuroradiol 1997;18: 135-143.

19 Baumgartner RW, Baumgartner I, Mattle HP, Schroth G: Transcranial color-coded duplex sonography in the evaluation of collateral flow through the circle of Willis. Am J Neuroradiol 1997; 18:127-133.

20 Fieschi C, Argentino C, Lenzi GL, Sacchetti ML, Toni D, Bozzao L: Clinical and instrumental evaluation of patients with ischemic stroke within the first six hours. J Neurol Sci 1989;91:311-321. 